\title{
The Raw Disk I/O Performance of Compaq Storage Works RAID Arrays under Tru64 UNIX
}

\author{
A.C. Uselton
}

\section{October 19, 2000}

U.S. Department of Energy

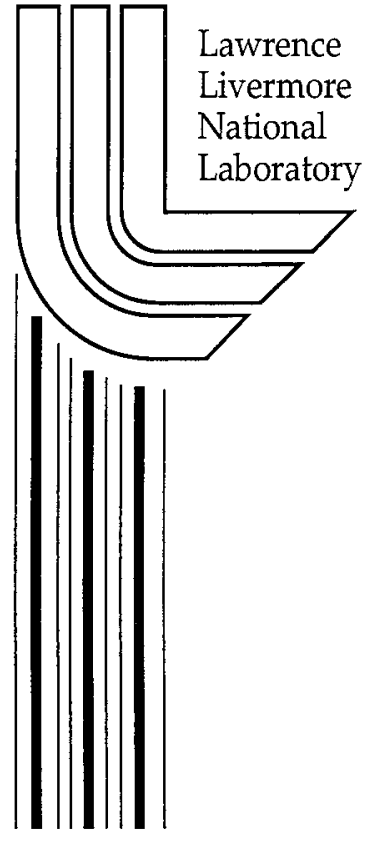




\section{DISCLAIMER}

This document was prepared as an account of work sponsored by an agency of the United States Government. Neither the United States Government nor the University of California nor any of their employees, makes any warranty, express or implied, or assumes any legal liability or responsibility for the accuracy, completeness, or usefulness of any information, apparatus, product, or process disclosed, or represents that its use would not infringe privately owned rights. Reference herein to any specific commercial product, process, or service by trade name, trademark, manufacturer, or otherwise, does not necessarily constitute or imply its endorsement, recommendation, or favoring by the United States Government or the University of California. The views and opinions of authors expressed herein do not necessarily state or reflect those of the United States Government or the University of California, and shall not be used for advertising or product endorsement purposes.

This work was performed under the auspices of the U.S. Department of Energy by the University of California, Lawrence Livermore National Laboratory under Contract No. W-7405-Eng-48.

This report has been reproduced

directly from the best available copy.

Available to DOE and DOE contractors from the

Office of Scientific and Technical Information

P.O. Box 62, Oak Ridge, TN 37831

Prices available from (423) 576-8401

http://apollo.osti.gov/bridge/

Available to the public from the

National Technical Information Service

U.S. Department of Commerce

5285 Port Royal Rd.,

Springfield, VA 22161

http://www.ntis.gov/

OR

Lawrence Livermore National Laboratory

Technical Information Department's Digital Library

http://www.llnl.gov/tid/Library.html 


\title{
The Raw Disk I/O Performance of Compaq StorageWorks RAID arrays under Tru64 UNIX
}

\author{
Andrew C. Uselton
}

October 19, 2000

\begin{abstract}
We report on the raw disk i/o performance of a set of Compaq StorageWorks RAID arrays connected to our cluster of Compaq ES40 computers via Fibre Channel. The best cumulative peak sustained data rate is $117 \mathrm{MB} / \mathrm{s}$ per node for reads and $77 \mathrm{MB} / \mathrm{s}$ per node for writes. This value occurs for a configuration in which a node has two Fibre Channel interfaces to a switch, which in turn has two connections to each of two Compaq StorageWorks RAID arrays. Each RAID array has two HSG80 RAID controllers controlling (together) two 5+p RAID chains. A $10 \%$ more space efficient arrangement using a single $11+\mathrm{p}$ RAID chain in place of the two $5+\mathrm{P}$ chains is $25 \%$ slower for reads and $40 \%$ slower for writes.
\end{abstract}

\section{Introduction}

This report presents the data transfer performance of the mass storage subsystem for a high performance computing system. All of the tests reported use unbuffered character data tranfers, or so-called raw $i / o$. The tests include several configurations of the storage subsytem in order to do the following: determine the optimum configuration, establish a baseline for further testing in conjunction with various file systems, and provide independent confirmation of the vendor's performance claims.

"I/o" is the generic term for input data transfer operations, or reads, and output data transfer operations, or writes. This report details values for the rate, in Megabytes per second (MB/s), at which data may be read from and written to the storage subsytem, and refers to such a value as a data rate. There are other measures for performance, including latency, reliablility or stability ${ }^{1}$, cpu utilization, price and capacity, but this report confines itself to data rate. Each i/o reads or writes a block of data whose size is measured in bytes. An i/o performance test consists of measuring the duration of one or more i/o operations of a given block size. The data rate varies depending on the configuration

\footnotetext{
${ }^{1}$ Both the "mean time between failures" and the ability to continue in the presence of errors.
} 


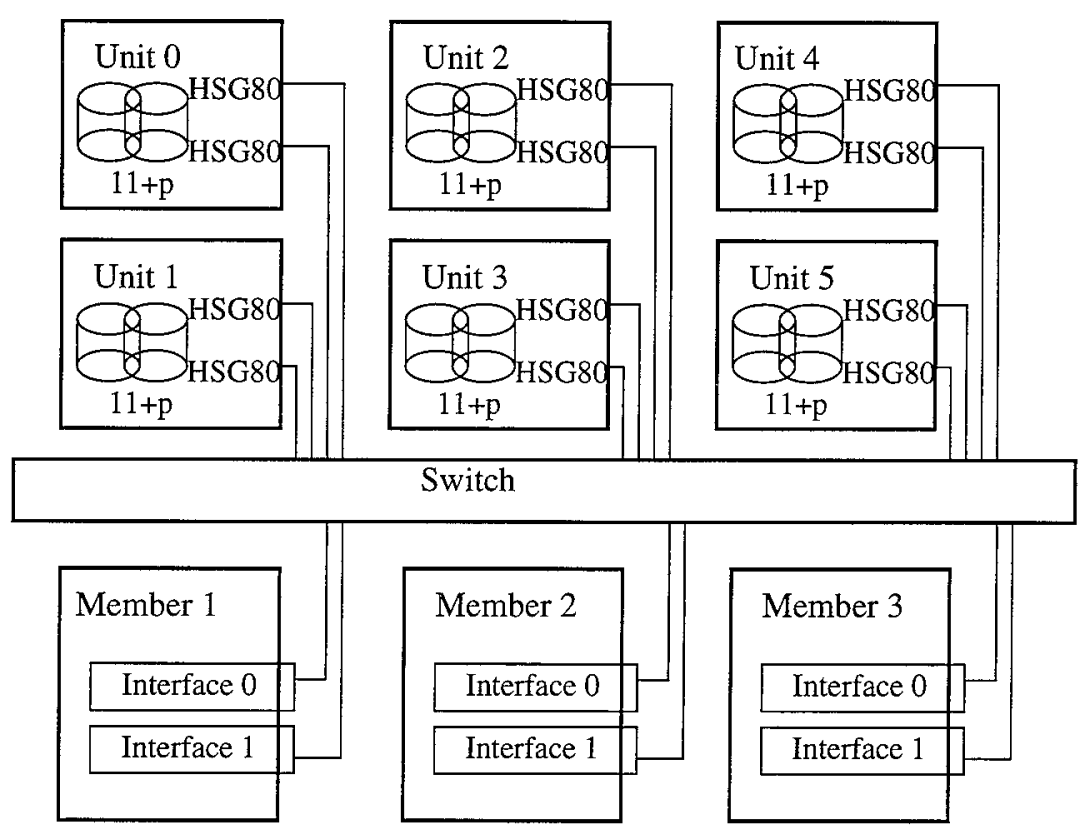

Figure 1: Test environment for clusters $\mathrm{A}$ and $\mathrm{B}$

of the storage subsystem and depending on the details of the $\mathrm{i} / \mathrm{o}$. The data rate assymptotically descends to a stable value as the total amount read or written becomes very large. The first tests ${ }^{2}$ varied the total amount of the $\mathrm{i} / \mathrm{o}$ in order to find the asymptotic value of data rate (the sustained data rate). In each case testing with the total amount of $i$ o four times the size of memory gives a good approximation of the sustained data rate. The reported tests vary the block size and show that the data rate improves with block size to a maximum for block sizes of $512 \mathrm{~KB}$ or larger.

The computer system being tested consists of 128 nodes connected via a high performance network interconnect. Each node is a Compaq ES40, which is an Alpha-based 4-way Symetric Multiprocessor (SMP) computer. The nodes are grouped into four clusters of 32 nodes each. The clusters are named cluster $A$, cluster $B$, cluster $C$, and cluster $D$. In each cluster each of the first three nodes, member 1, member 2 , and member 3, is connected to a set of Compaq StorageWorks RAID arrays and is referred to as an $i / o$ node. The clusters are running Compaq's Sierra cluster software based on the Tru64 UNIX operaing system. In each cluster members 1 and 2 each has 8 Gigabytes (GB) of memory while members 3 through 32 each has 2 GB of memory.

Figure 1 shows the layout of the i/o nodes and the RAID arrays for clusters A and B. The connection between i/o nodes and RAID arrays is via Fibre Channel, which can transfer $100 M B$ per second. The figure shows each of the $i / o$ nodes with two Fibre Channel interfaces connecting to a Fibre Channel switch. The switch is connected via Fibre Channel to each of two HSG80 RAID controllers on each RAID array. There are six RAID arrays in each cluster, and they are

\footnotetext{
${ }^{2}$ Only reported in the appendix.
} 

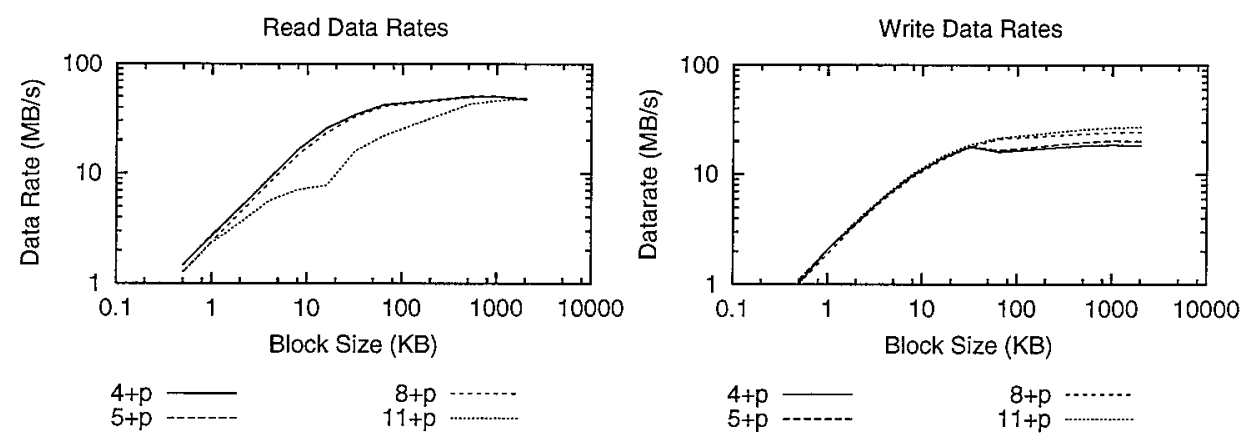

Figure 2: Read and Write data rates for single unit i/o

labelled Unit 0 through Unit 5. In Figure 1 each RAID array has a set of twelve SCSI disks in a RAID-5 configuration with one disk acting as a parity disk. We refer to this as an $11+p$ RAID chain. The only change in clusters $C$ and D is that each RAID array is configured to have two $5+$ p RAID chains in place of one $11+\mathrm{p}$ RAID chain.

The remainder of this report consists of four sections each of which details the results of a particular group of tests. In Section 2 the data rate varies with the size of the RAID chain. In Section 3 the data rate of two $5+p$ RAID chains is $60 \%$ better for reads over a single $11+$ p RAID chain and $40 \%$ better for writes at the expense of $10 \%$ of the available storage space. In Section 4 the data rate when using two RAID arrays via a single (member) interface is about double that of one, but using three RAID arrays increases the data rate very little. Similarly, in Section 5 using two interfaces (to access two or more RAID arrays) about doubles the data rate. This report concludes with a summary of the results, a reccomendation for the storage subsystem's configuration, and some suggestions for further research. The author reccommends that each RAID array be configured with two equal sized RAID chains, each as large as the RAID array can contain. In such a configuration each node should be able to sustain at least a $117 \mathrm{MB} / \mathrm{s}$ read data rate and a $77 \mathrm{MB} / \mathrm{s}$ write data rate. An appendix discusses methodology.

\section{The size of the RAID chain}

Figure 2 graphs the read and write data rates against block size for four configurations of RAID chain: $4+\mathrm{p}, 5+\mathrm{p}, 8+\mathrm{p}$, and $11+\mathrm{p}$. Each test was conducted 
on member 2 of one of the clusters, and the i/o was directed through a single Fibre Channel interface to a single RAID chain in a single RAID array. In each case the node has $2 \mathrm{~GB}$ of memory and the sustained data rate was measured at a value for total i/o of $8 \mathrm{~GB}$. Each curve represents a set of tests each conducted with block size set to one of $512 B, 1024 B(=1 K B), 2 K B, 4 K B, 8 K B, 16 K B$, $32 K B, 64 K B, 512 K B, 1024 K B(=1 M B)$, and $2 M B$.

The read data rate curves for $4+\mathrm{p}, 5+\mathrm{p}$, and $8+\mathrm{p}$, are nearly identical and show two prominent features. First, when the block size is $512 K B$ or larger the read data rate is in the vicinity of $50 \mathrm{MB} / \mathrm{s}$ which appears to be the peak sustained read data rate a single RAID chain can produce regardles of the number of disks in the chain. Second, for block sizes $16 K B$ and smaller (a log plot of) the data rate is linear with block size. The later point deserves some elaboration.

If data rate is linear in block size then the time for an i/o operation is constant, independent of block size. The explanation is as follows: Let $r$ represent the data rate, $b$ the block size, $n$ the number of blocks read, $t$ the total amount read, and $s$ the time it took to read $t$. Then

$$
r=t / s=b * n / s
$$

If $r$ is a linear function of $b$ then $n / s$ is constant, which is to say that the number of blocks read per second does not change as $b$ changes. If the data rate were linear in block size then the slope of the curve in the log plot would be exactly one:

$$
r=m b \rightarrow \log (r)=\log (m b)=\log (m)+\log (b)
$$

In fact the slope is approximately 1.1 :

$$
1.1 \log (b)=\log \left(b^{1.1}\right)
$$

Which leaves us to wonder why $n / s \sim b^{0.1}$. In any case the RAID chain can produce at most $50 \mathrm{MB} / \mathrm{s}$ and no more than $3000 \mathrm{blocks} / \mathrm{s}$ at best.

The $11+\mathrm{p}$ configuration of RAID chain has generally similar limitting behaviors, but shows a remarkable drop in data rate for medium sized blocks. It looks like the sum of two distinct asymptotic behaviors. One may wonder what is happenning that does not happen for the other RAID chains.

The write data rate curves are similar for all four configurations of RAID chain. For block sizes of $32 K B$ and less the behaviors are identical, with an assymptotic increase of data rate to a maximum near $20 \mathrm{MB} / \mathrm{s}$. Above $32 \mathrm{~KB}$ the $11+p$ curve continues smoothly to the highest asymptotic value of $27 \mathrm{MB} / \mathrm{s}$. For large block sizes the smaller RAID chains show smaller peak values. On may speculate that the $11+\mathrm{p}$ write data rate curve is a limitting case for StorageWorks RAID chains.

\section{The number of chains in a RAID array}

Figure 3 again shows the read and write data rates for the $11+p$ RAID chain as well as an arrangement in which the same twelve disks are configured in 

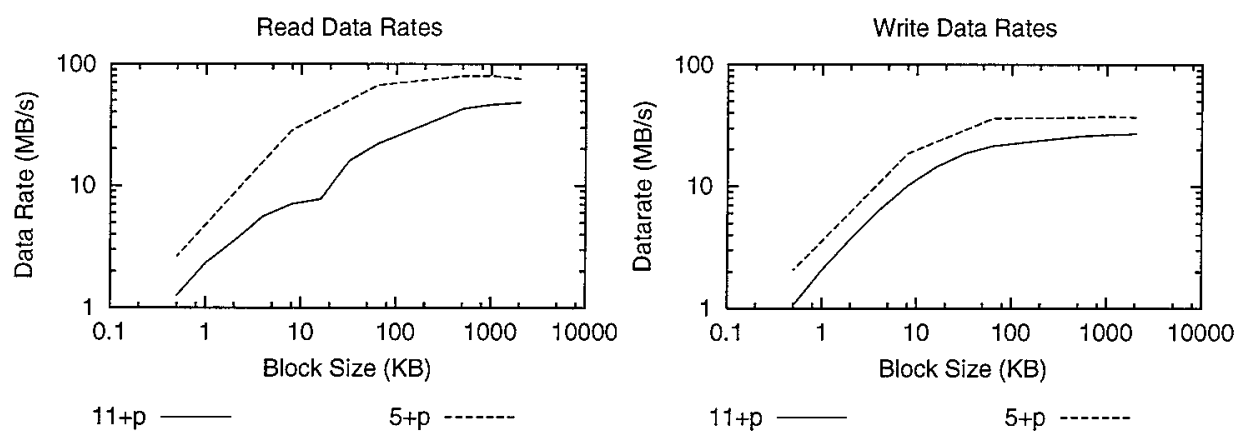

Figure 3: Twelve disks configured as one $11+\mathrm{p}$ or two $5+\mathrm{p}$ chains

the RAID array as two separate RAID chains. The benefit of doing so is that both HSG80s can be active at once servicing i/o operations. The result is a $60 \%$ increase in read data rate and a $36 \%$ increase in write data rate. In fact the read data rate is near the theoretical maximum for SCSI transfers. The write rate for the two $5+\mathrm{p}$ chains is nearly double the write rate for one, so we may speculate that two larger RAID chains might perform even better. The advantage of the $11+p$ RAID chain is that it has $10 \%$ more space than the two $5+\mathrm{p}$ chains.

\section{The number of RAID arrays}

In Figures 4 and 5 the graphs show the data rate for a single node using a single Fibre Channel interface reading from and writing to one, two, and three $11+\mathrm{p}$ chains and (respectively) two, four, and six $5+\mathrm{p}$ chains in one, two, and three units.

The read and write data rates for the $11+\mathrm{p}$ chains improve when writing to two units as does the write data rate for the $5+\mathrm{p}$ chains. Once the read data rate from a node reaches around $80 \mathrm{MB} / \mathrm{s}$ in cannot be improved upon by adding more units to the node's interface. Similarly, a node seems to be unable to write more than $50 \mathrm{MB} / \mathrm{s}$ through a single interface no matter how many units it can write to. It is clear that if performance is an issue then a single interface should not be given more than two units for its i/o resposibilities. 

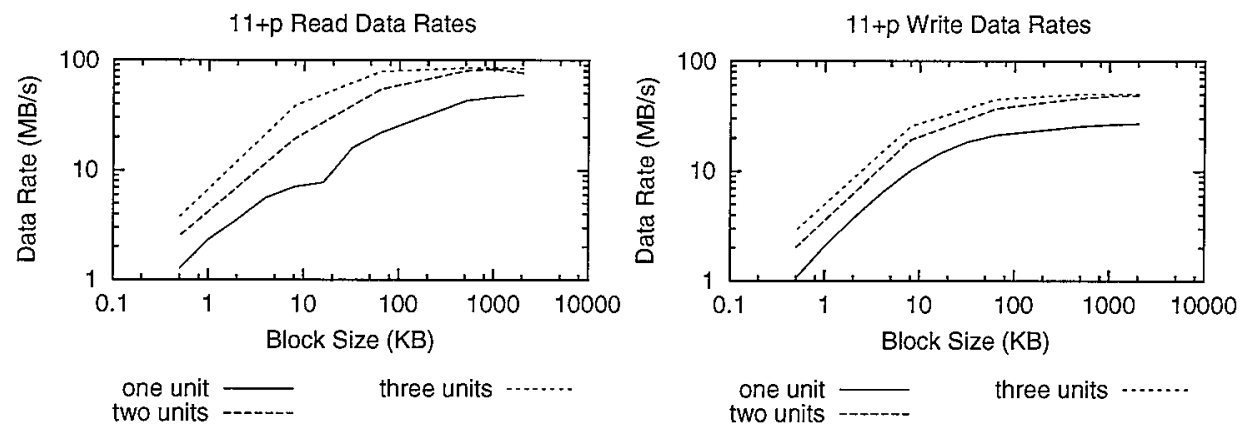

Figure 4: $\mathrm{I} / \mathrm{o}$ to multiple $11+\mathrm{p}$ units via a single interface
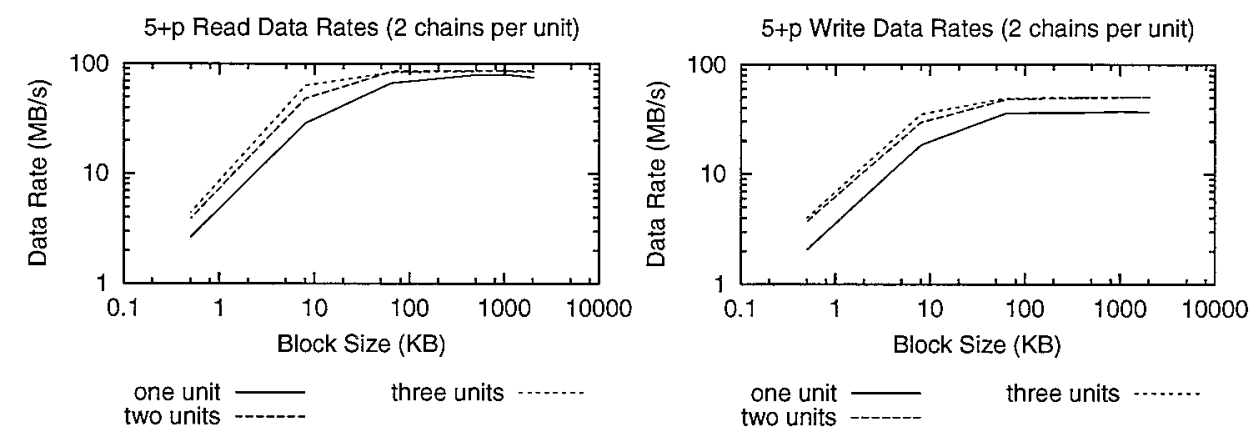

Figure 5: I/o to multiple 5+p units (two chains each) via a single inteface 

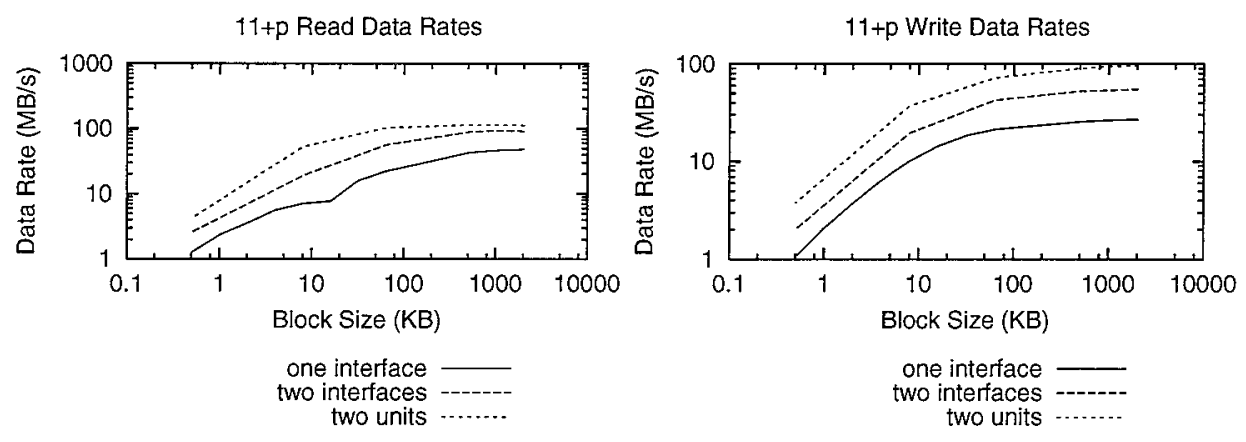

Figure 6: $1 / 0$ to two $11+p$ units via two interfaces

\section{The number of interfaces}

Figures 6 and 7 show the preformance gain from using two interfaces on a single node. In each graph there is the curve already presented in which a single interface communicates with a single unit (either configured with an $11+p$ chian as in Figure 6 or two $5+$ p chains as in Figure 7). Next, each graph shows a curve for the read and write data rates when employing two interfaces. And finally, there is a curve for the performance of $i / o$ to two units on each of two interfaces.

The read and write data rates for using two $11+\mathrm{p}$ RAID chains on two interfaces double those for a single unit, as does the write data rate for the $5+\mathrm{p}$ chains double. The read data rate increases by about $50 \%$ to $115 \mathrm{MB} / \mathrm{s}$. For both the $11+\mathrm{p}$ and the $5+\mathrm{p}$ configurations the peak read data rate is $114 \mathrm{MB} / \mathrm{s}$ and the peak write data rate near $100 \mathrm{MB} / \mathrm{s}$. Tests with three units on each interface show no improvement. We may speculate that a third interface would not increase the read data rate, since it is not increasing with the addition of a second or third unit to the interface whereas a second unit did improve the performance when only one interface was employed. On the other hand the write data rate for two interfaces always doubled the data rate for the same configuration with one interface, so a third interface might still improve the write performance. 

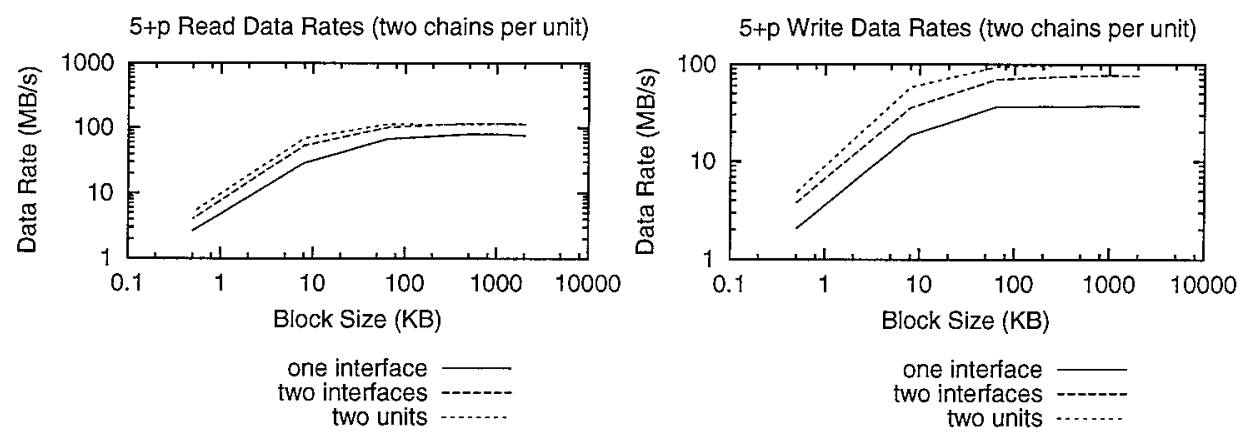

Figure 7: I/o to two $5+\mathrm{p}$ units (two chains per unit) via two intefaces

\section{Conclusion}

Current plans for this computer system are for it to have three i/o nodes communicating with six StorageWorks units, and for the entire storage subsystem to be gathered into a single large parallel file system. If each of the i/o nodes is configured with one unit on each interface and if each unit is configured with two $5+$ p RAID chains then the cumulative peak sustained data rate for the storage subsystem is (in theory) $351 \mathrm{MB} / \mathrm{s}$ for reads and $231 \mathrm{MB} / \mathrm{s}$ for writes. This is the configuration that the author recommends. A configuration using $11+\mathrm{p}$ RAID chains could achieve $282 \mathrm{MB} / \mathrm{s}$ for reads and $165 \mathrm{MB} / \mathrm{s}$ for writes, and would have $10 \%$ more file space. An altered arrangement (with more units) in which two units were on each interface and only two i/o nodes were used could achieve $228 \mathrm{MB} / \mathrm{s}$ for reads and $200 \mathrm{MB} / \mathrm{s}$ for writes.

This report leaves a few questions unanswered. Some questions will be addressed in a second report in which file systems are used for the i/o rather than raw i/o. It would be interesting to determine what causes the dip in read data rate for $11+\mathrm{p}$ RAID chains. The StorageWorks RAID arrays have room for a total of 24 SCSI disks, so there might be an improvement in data rate for a configuration with two RAID chains of $11+p$ each in a single unit. All three of the i/o nodes can see all six of the StorageWorks units, so a test with all nodes writing to one unit would better determine the maximum performance of a single unit. 

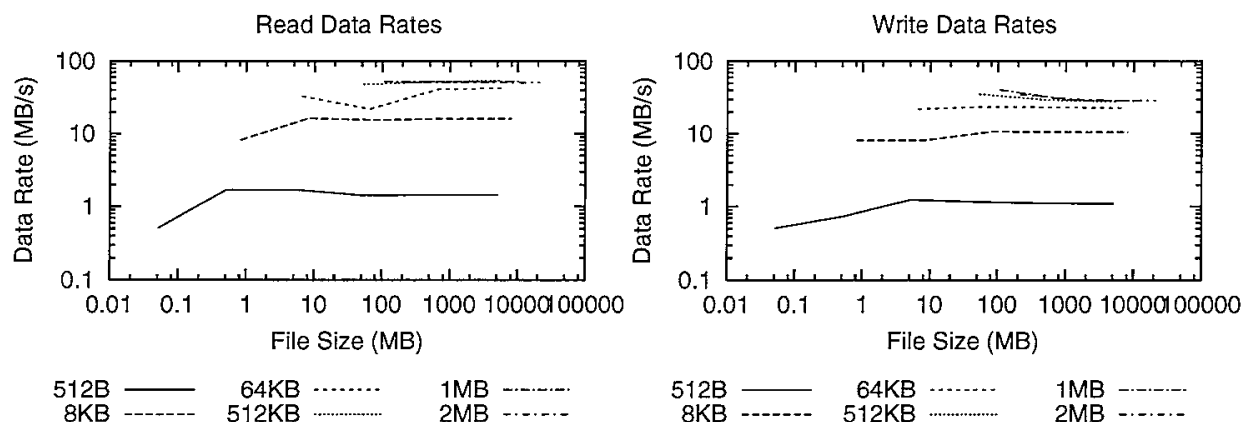

Figure 8: Data rate asymptotes for large files

\section{A Methodology}

All of the tests in this report followed the same basic scheme. In a UNIX shell the $d d$ (disk duplicate) command copies from one location and to another. The $d d$ command is invoked within a time command. The result is an estimate, acurate to about $1 / 10$ second of the time necesary to perform the copy.

The $d d$ command requires both in input file and an output file. When measuring read data rate one wants to be certain that the time spent writing is negligable. Conversely, when measuring write data rate one wants the time spent reading to be negligable. The "devices" /dev/zero and /dev/null perform this function. A degenerate case in which $d d$ both reads from/dev/zero and writes to /dev/null has a data rate one to two orders of magnitude higher than when accessing an actual device. Thus a command like

time dd if $=/ \mathrm{dev} / \mathrm{rdisk} / \mathrm{dsk} 10 \mathrm{c}$ of $=/ \mathrm{dev} /$ null

is a fair test of the read performance of $d s k 10 c$.

While all the tests in this report use raw disks with no buffering, there is always the possibility that some buffering is taking place in the RAID array itself. Figure 8 shows the data rate for performing the i/o tests with successively larger files and with various block sizes. The asymptotic behavior above one or two GB written converges to a value characteristic for the block size.

For the smallest files represented in Figure 8 the total amount of time spent reading or writing was comparable with the $1 / 10$ second resolution of the time command. All of the tests shown in the main body of this report ran for more than 60 seconds, so the resolution of the time command was not a problem. 

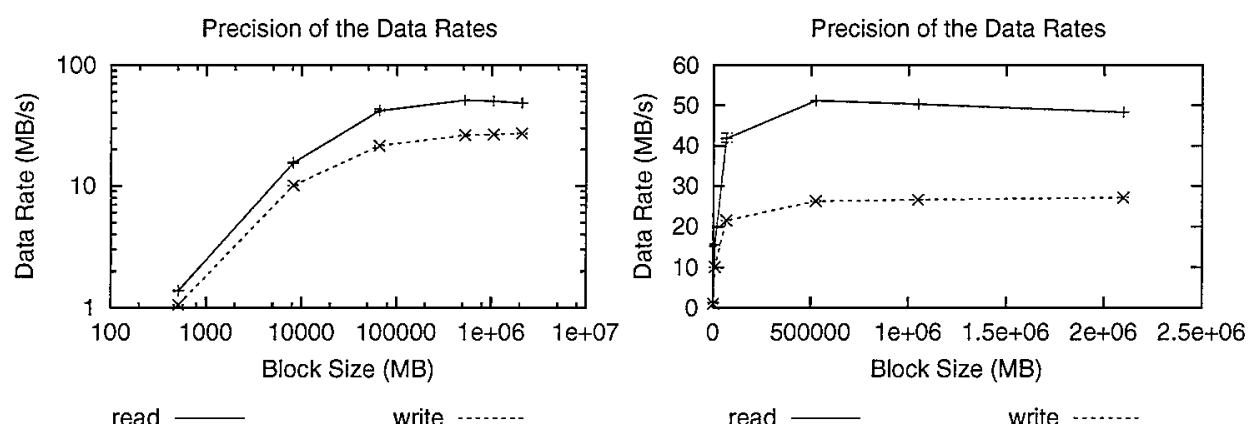

Figure 9: The measuments are precise to within $5 \%$.

Error could enter into the observations from other sources as well, so one may wonder what the precision of the measurments is. Figure 9 shows the results of running tests for each data point five times and putting error bars around the extreme values. In every case the difference delta between the highest observed value and the lowest is less than $5 \%$ of the magnitude of the measurement. The log plot makes the error bars difficult to see so the same graph is shown without $\log$ scales on the right. Even here the error bars are so small they are difficult to see on the graph.

The foregoing characterizes the precision of the measurment process. That is, repeated tests are close together - within $5 \%$. An alternative means of measuring the same values is available in the Ronnie benchmark. Figure 10 shows the results of running Ronnie on a $4+p$ configured RAID array and writing to a single chain with $1 \mathrm{MB}$ blocks. The data rate for running one Ronnie job is $15 \mathrm{MB} / \mathrm{s}$, around $20 \%$ less than the $18 \mathrm{MB} / \mathrm{s}$ computed using time and $d d$. The accuracy of the methods presented should be taken as no better than $20 \%$. Note that adding a second parallel Ronnie job doubles the data rate, but additional jobs reduce the data rate back to arond $24 \mathrm{MB} / \mathrm{s}$.

Also shown in Figure 10 are the values for CPU utilization while running Ronnie. A single Ronnie job consumes about $0.2 \%$ of the capacity of a node. This is in keeping with the values top reported when running a test with $d d$. That is, none of the tests reported here put much load on the nodes' CPUs.

\section{B Sample Scripts}

Here is a typical script for invoking time and $d d$. 


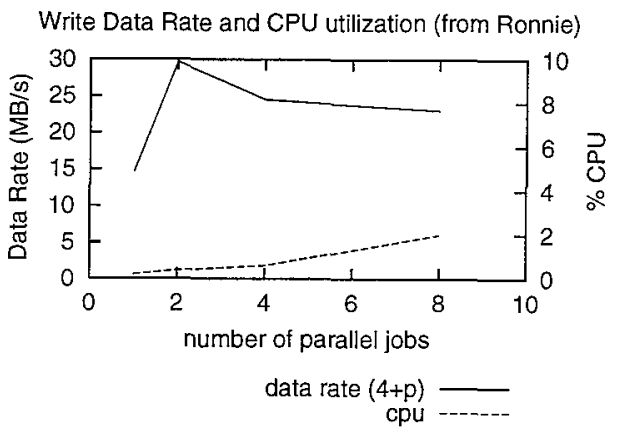

Figure 10: The measurements are accurate within about $20 \%$.

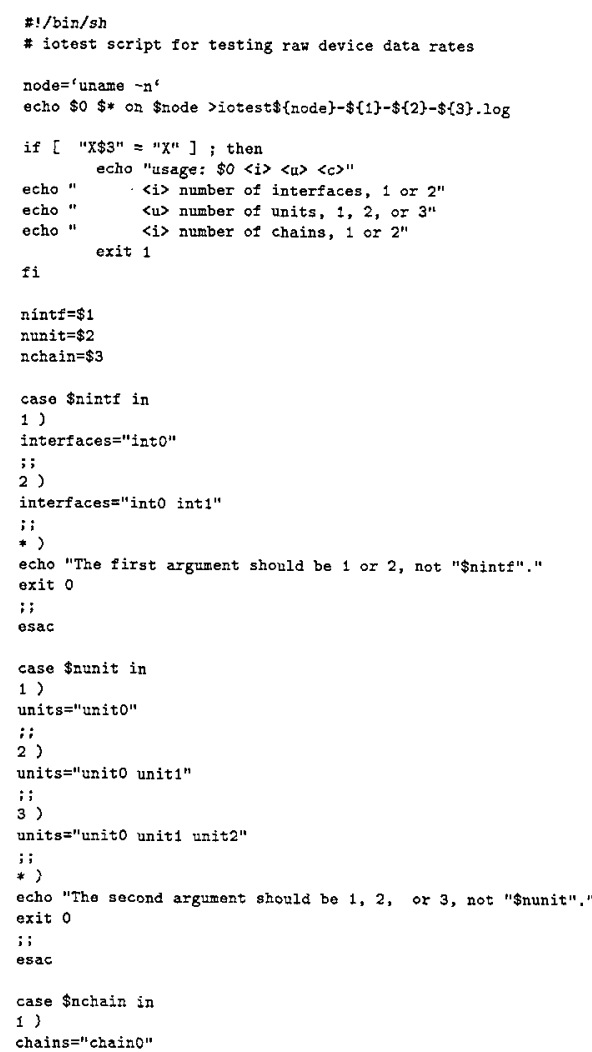




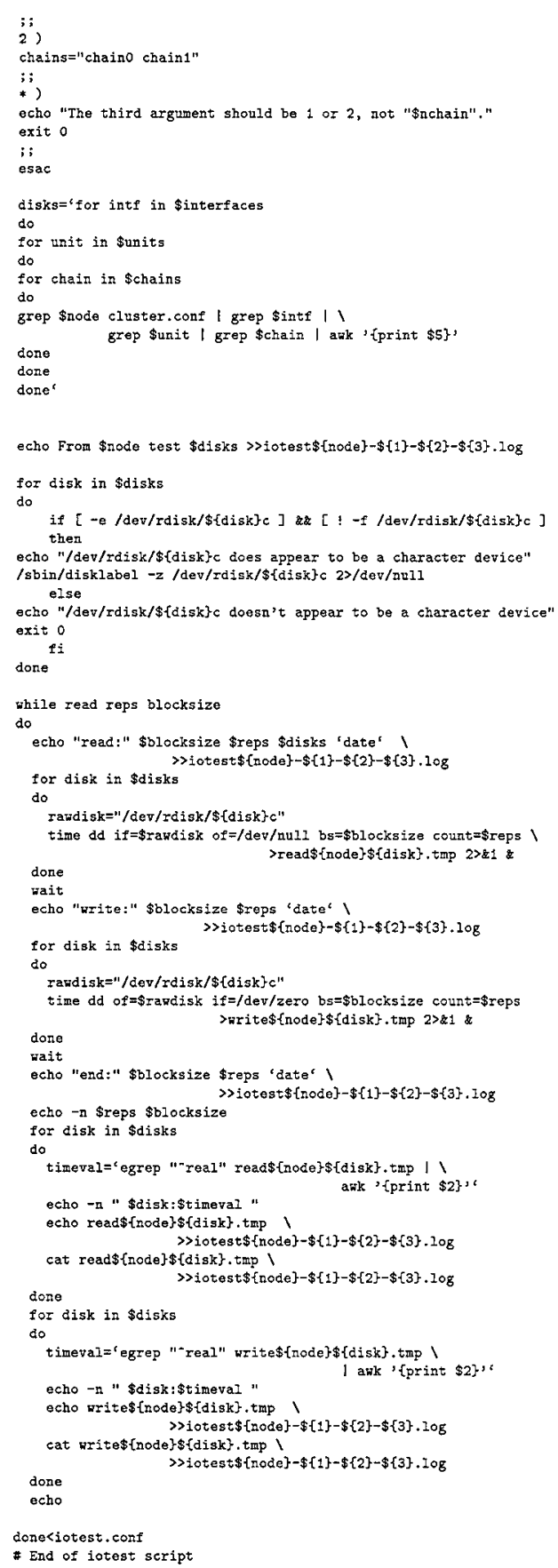

The script uses $s h$ which relies on /usr/bin/time rather than a built in function. On the other hand, wait is a built in and is used here, since /usr/bin/wait 
does not behave as one might expect ${ }^{3}$.

The script produces a series of times, which a separate filter then turns into data rates in files with the "data" extension. The following typical gnuplot script generates a graph to be included in this paper (".eps") or on a web page (".gif"). This one generates the graph on the left in Figure 2.

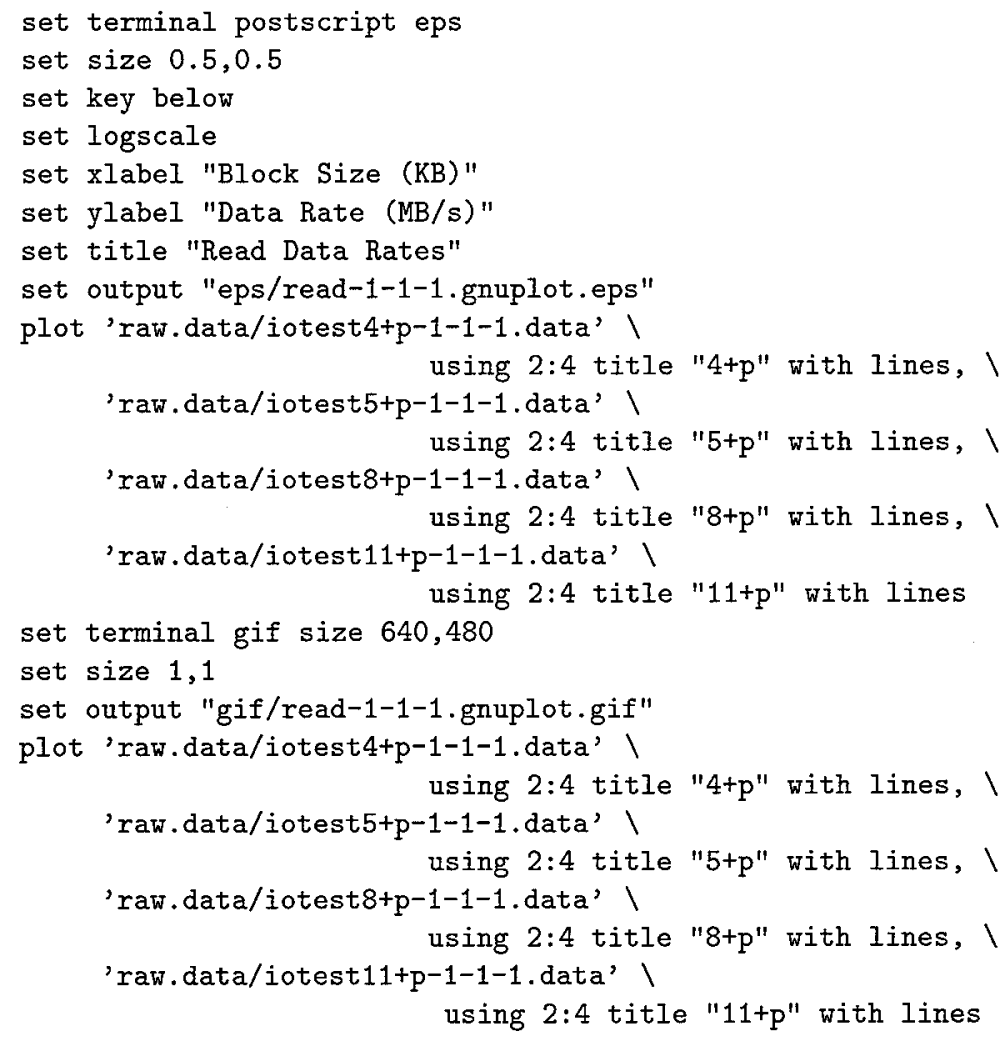

\section{Data}

The tables in Figures 11 through 27 show the contents of the".data" files from which all of the graphs were constructed.

\footnotetext{
${ }^{3}$ It creates a subprocess that waits only for itself, thus it return immediately
} 


\begin{tabular}{|c|c|c|c|c|}
\hline count & $\begin{array}{c}\text { block size } \\
(K B)\end{array}$ & $\begin{array}{c}\text { amount written } \\
(M B)\end{array}$ & $\begin{array}{c}\text { read rate } \\
(M B / s)\end{array}$ & $\begin{array}{c}\text { write rate } \\
(M B / s)\end{array}$ \\
\hline 4096 & 2048 & 8192 & 47.1 & 18.2 \\
\hline 8192 & 1024 & 8192 & 49.9 & 18.6 \\
\hline 16384 & 512 & 8192 & 49.9 & 18.3 \\
\hline 131072 & 64 & 8192 & 42.4 & 15.9 \\
\hline 262144 & 32 & 8192 & 34.3 & 17.8 \\
\hline 524288 & 16 & 8192 & 25.9 & 13.8 \\
\hline 1048576 & 8 & 8192 & 16.4 & 9.8 \\
\hline 2097152 & 4 & 8192 & 9.0 & 6.2 \\
\hline 4194304 & 2 & 8192 & 4.9 & 3.6 \\
\hline 8388608 & 1 & 8192 & 2.7 & 2.1 \\
\hline 16777216 & 0.5 & 8192 & 1.5 & 1.0 \\
\hline
\end{tabular}

Figure 11: A single 4+p RAID chain

\begin{tabular}{|c|c|c|c|c|}
\hline count & $\begin{array}{c}\text { block size } \\
(K B)\end{array}$ & $\begin{array}{c}\text { amount written } \\
(M B)\end{array}$ & $\begin{array}{c}\text { read rate } \\
(M B / s)\end{array}$ & $\begin{array}{c}\text { write rate } \\
(M B / s)\end{array}$ \\
\hline 4096 & 2048 & 8192 & 48.1 & 20.0 \\
\hline 8192 & 1024 & 8192 & 50.2 & 20.2 \\
\hline 16384 & 512 & 8192 & 50.4 & 19.5 \\
\hline 131072 & 64 & 8192 & 42.2 & 16.4 \\
\hline 262144 & 32 & 8192 & 34.3 & 17.9 \\
\hline 524288 & 16 & 8192 & 25.5 & 13.9 \\
\hline 1048576 & 8 & 8192 & 16.3 & 9.8 \\
\hline 2097152 & 4 & 8192 & 8.9 & 6.2 \\
\hline 4194304 & 2 & 8192 & 4.9 & 3.7 \\
\hline 8388608 & 1 & 8192 & 2.7 & 2.1 \\
\hline 16777216 & $1 / 2$ & 8192 & 1.5 & 1.1 \\
\hline
\end{tabular}

Figure 12: A single 5+p RAID chain 


\begin{tabular}{|c|c|c|c|c|}
\hline count & $\begin{array}{c}\text { block size } \\
(K B)\end{array}$ & $\begin{array}{c}\text { amount written } \\
(M B)\end{array}$ & $\begin{array}{c}\text { read rate } \\
(M B / s)\end{array}$ & $\begin{array}{c}\text { write rate } \\
(M B / s)\end{array}$ \\
\hline 4096 & 2048 & 8192 & 47.0 & 24.4 \\
\hline 8192 & 1024 & 8192 & 50.6 & 24.1 \\
\hline 16384 & 512 & 8192 & 49.6 & 23.4 \\
\hline 131072 & 64 & 8192 & 41.0 & 21.0 \\
\hline 262144 & 32 & 8192 & 33.1 & 17.6 \\
\hline 524288 & 16 & 8192 & 23.6 & 13.4 \\
\hline 1048576 & 8 & 8192 & 14.7 & 9.4 \\
\hline 2097152 & 4 & 8192 & 8.2 & 6.0 \\
\hline 4194304 & 2 & 8192 & 4.4 & 3.5 \\
\hline 8388608 & 1 & 8192 & 2.4 & 1.9 \\
\hline 16777216 & $1 / 2$ & 8192 & 1.3 & 1.0 \\
\hline
\end{tabular}

Figure 13: A single 8+p RAID chain

\begin{tabular}{|c|c|c|c|c|}
\hline count & $\begin{array}{c}\text { block size } \\
(K B)\end{array}$ & $\begin{array}{c}\text { amount written } \\
(M B)\end{array}$ & $\begin{array}{c}\text { read rate } \\
(M B / s)\end{array}$ & $\begin{array}{c}\text { write rate } \\
(M B / s)\end{array}$ \\
\hline 4096 & 2048 & 8192 & 48.2 & 27.0 \\
\hline 8192 & 1024 & 8192 & 46.3 & 26.5 \\
\hline 16384 & 512 & 8192 & 43.0 & 25.7 \\
\hline 131072 & 64 & 8192 & 22.0 & 21.4 \\
\hline 262144 & 32 & 8192 & 15.9 & 18.6 \\
\hline 524288 & 16 & 8192 & 7.8 & 14.4 \\
\hline 1048576 & 8 & 8192 & 7.1 & 10.1 \\
\hline 2097152 & 4 & 8192 & 5.6 & 6.4 \\
\hline 4194304 & 2 & 8192 & 3.6 & 3.7 \\
\hline 8388608 & 1 & 8192 & 2.3 & 2.1 \\
\hline 16777216 & $1 / 2$ & 8192 & 1.3 & 1.1 \\
\hline
\end{tabular}

Figure 14: A single 11+p RAID chain

\begin{tabular}{|c|c|c|c|c|}
\hline count & $\begin{array}{c}\text { block size } \\
(K B)\end{array}$ & $\begin{array}{c}\text { amount written } \\
(M B)\end{array}$ & $\begin{array}{c}\text { read rate } \\
(M B / s)\end{array}$ & $\begin{array}{c}\text { write rate } \\
(M B / s)\end{array}$ \\
\hline 5000 & 2048 & 10000 & 75.3 & 36.8 \\
\hline 10000 & 1024 & 10000 & 79.9 & 37.4 \\
\hline 20000 & 512 & 10000 & 79.8 & 36.7 \\
\hline 150000 & 64 & 9375 & 67.0 & 36.2 \\
\hline 600000 & 8 & 4688 & 28.7 & 18.5 \\
\hline 1000000 & $1 / 2$ & 488 & 2.7 & 2.1 \\
\hline
\end{tabular}

Figure 15: Two 5+p RAID chains in one RAID array 


\begin{tabular}{|c|c|c|c|c|}
\hline count & $\begin{array}{c}\text { block size } \\
(K B)\end{array}$ & $\begin{array}{c}\text { amount written } \\
(M B)\end{array}$ & $\begin{array}{c}\text { read rate } \\
(M B / s)\end{array}$ & $\begin{array}{c}\text { write rate } \\
(M B / s)\end{array}$ \\
\hline 5000 & 2048 & 10000 & 76.2 & 49.1 \\
\hline 10000 & 1024 & 10000 & 83.7 & 48.2 \\
\hline 20000 & 512 & 10000 & 80.7 & 46.2 \\
\hline 150000 & 64 & 9375 & 54.3 & 37.0 \\
\hline 600000 & 8 & 4688 & 19.5 & 19.3 \\
\hline 1000000 & $1 / 2$ & 488 & 2.5 & 2.0 \\
\hline
\end{tabular}

Figure 16: Two 11+p RAID chains in two RAID arrays via one interface

\begin{tabular}{|c|c|c|c|c|}
\hline count & $\begin{array}{c}\text { block size } \\
(K B)\end{array}$ & $\begin{array}{c}\text { amount written } \\
(M B)\end{array}$ & $\begin{array}{c}\text { read rate } \\
(M B / s)\end{array}$ & $\begin{array}{c}\text { write rate } \\
(M B / s)\end{array}$ \\
\hline 5000 & 2048 & 10000 & 84.4 & 50.5 \\
\hline 10000 & 1024 & 10000 & 85.7 & 50.3 \\
\hline 20000 & 512 & 10000 & 85.4 & 50.1 \\
\hline 150000 & 64 & 9375 & 78.8 & 45.1 \\
\hline 600000 & 8 & 4688 & 38.6 & 25.6 \\
\hline 1000000 & $1 / 2$ & 488 & 3.8 & 2.9 \\
\hline
\end{tabular}

Figure 17: Three 11+p RAID chains in three RAID arrays via one interface

\begin{tabular}{|c|c|c|c|c|}
\hline count & $\begin{array}{c}\text { block size } \\
(K B)\end{array}$ & $\begin{array}{c}\text { amount written } \\
(M B)\end{array}$ & $\begin{array}{c}\text { read rate } \\
(M B / s)\end{array}$ & $\begin{array}{c}\text { write rate } \\
(M B / s)\end{array}$ \\
\hline 5000 & 2048 & 10000 & 85.6 & 50.5 \\
\hline 10000 & 1024 & 10000 & 86.8 & 50.3 \\
\hline 20000 & 512 & 10000 & 86.9 & 50.3 \\
\hline 150000 & 64 & 9375 & 85.0 & 48.2 \\
\hline 600000 & 8 & 4688 & 48.3 & 29.8 \\
\hline 1000000 & $1 / 2$ & 488 & 3.9 & 3.7 \\
\hline
\end{tabular}

Figure 18: Two 5+p RAID chains in each of two RAID arrays via one interface

\begin{tabular}{|c|c|c|c|c|}
\hline count & $\begin{array}{c}\text { block size } \\
(K B)\end{array}$ & $\begin{array}{c}\text { amount written } \\
(M B)\end{array}$ & $\begin{array}{c}\text { read rate } \\
(M B / s)\end{array}$ & $\begin{array}{c}\text { write rate } \\
(M B / s)\end{array}$ \\
\hline 5000 & 2048 & 10000 & 85.6 & 50.6 \\
\hline 10000 & 1024 & 10000 & 85.6 & 50.5 \\
\hline 20000 & 512 & 10000 & 85.4 & 50.4 \\
\hline 150000 & 64 & 9375 & 83.7 & 49.6 \\
\hline 600000 & 8 & 4688 & 63.6 & 35.2 \\
\hline 1000000 & $1 / 2$ & 488 & 4.4 & 4.0 \\
\hline
\end{tabular}

Figure 19: Two 5+p RAID chains in each of three RAID arrays via one interface 


\begin{tabular}{|c|c|c|c|c|}
\hline count & $\begin{array}{c}\text { block size } \\
(K B)\end{array}$ & $\begin{array}{c}\text { amount written } \\
(M B)\end{array}$ & $\begin{array}{c}\text { read rate } \\
(M B / s)\end{array}$ & $\begin{array}{c}\text { write rate } \\
(M B / s)\end{array}$ \\
\hline 5000 & 2048 & 10000 & 91.4 & 55.1 \\
\hline 10000 & 1024 & 10000 & 94.0 & 53.8 \\
\hline 20000 & 512 & 10000 & 89.4 & 52.6 \\
\hline 150000 & 64 & 9375 & 56.5 & 42.5 \\
\hline 600000 & 8 & 4688 & 18.9 & 19.6 \\
\hline 1000000 & $1 / 2$ & 488 & 2.6 & 2.0 \\
\hline
\end{tabular}

Figure 20: One 11+p RAID chain in each of two RAID arrays via two interfaces

\begin{tabular}{|c|c|c|c|c|}
\hline count & $\begin{array}{c}\text { block size } \\
(K B)\end{array}$ & $\begin{array}{c}\text { amount written } \\
(M B)\end{array}$ & $\begin{array}{c}\text { read rate } \\
(M B / s)\end{array}$ & $\begin{array}{c}\text { write rate } \\
(M B / s)\end{array}$ \\
\hline 5000 & 2048 & 10000 & 112.7 & 95.6 \\
\hline 10000 & 1024 & 10000 & 113.8 & 93.9 \\
\hline 20000 & 512 & 10000 & 114.0 & 90.8 \\
\hline 150000 & 64 & 9375 & 103.5 & 71.9 \\
\hline 600000 & 8 & 4688 & 52.9 & 37.1 \\
\hline 1000000 & $1 / 2$ & 488 & 4.2 & 3.8 \\
\hline
\end{tabular}

Figure 21: One 11+p RAID chain in each of four RAID arrays via two interfaces

\begin{tabular}{|c|c|c|c|c|}
\hline count & $\begin{array}{c}\text { block size } \\
(K B)\end{array}$ & $\begin{array}{c}\text { amount written } \\
(M B)\end{array}$ & $\begin{array}{c}\text { read rate } \\
(M B / s)\end{array}$ & $\begin{array}{c}\text { write rate } \\
(M B / s)\end{array}$ \\
\hline 5000 & 2048 & 10000 & 118.5 & 89.2 \\
\hline 10000 & 1024 & 10000 & 118.6 & 88.5 \\
\hline 20000 & 512 & 10000 & 118.5 & 87.6 \\
\hline 150000 & 64 & 9375 & 112.0 & 80.3 \\
\hline 600000 & 8 & 4688 & 68.7 & 47.3 \\
\hline 1000000 & $1 / 2$ & 488 & 4.4 & 4.0 \\
\hline
\end{tabular}

Figure 22: One $11+\mathrm{p}$ RAID chain in each of six RAID arrays via two interfaces

\begin{tabular}{|c|c|c|c|c|}
\hline count & $\begin{array}{c}\text { block size } \\
(K B)\end{array}$ & $\begin{array}{c}\text { amount written } \\
(M B)\end{array}$ & $\begin{array}{c}\text { read rate } \\
(M B / s)\end{array}$ & $\begin{array}{c}\text { write rate } \\
(M B / s)\end{array}$ \\
\hline 5000 & 2048 & 10000 & 114.2 & 76.0 \\
\hline 10000 & 1024 & 10000 & 116.7 & 76.7 \\
\hline 20000 & 512 & 10000 & 117.6 & 75.9 \\
\hline 150000 & 64 & 9375 & 101.2 & 69.4 \\
\hline 600000 & 8 & 4688 & 53.1 & 35.6 \\
\hline 1000000 & 0 & 488 & 4.0 & 3.8 \\
\hline
\end{tabular}

Figure 23: Two 5+p RAID chains in each of two RAID arrays via two interfaces 


\begin{tabular}{|c|c|c|c|c|}
\hline count & $\begin{array}{c}\text { block size } \\
(K B)\end{array}$ & $\begin{array}{c}\text { amount written } \\
(M B)\end{array}$ & $\begin{array}{c}\text { read rate } \\
(M B / s)\end{array}$ & $\begin{array}{c}\text { write rate } \\
(M B / s)\end{array}$ \\
\hline 5000 & 2048 & 10000 & 114.4 & 99.8 \\
\hline 10000 & 1024 & 10000 & 114.5 & 99.3 \\
\hline 20000 & 512 & 10000 & 114.4 & 99.1 \\
\hline 150000 & 64 & 9375 & 113.6 & 94.2 \\
\hline 600000 & 8 & 4688 & 68.5 & 57.8 \\
\hline 1000000 & $1 / 2$ & 488 & 5.1 & 4.8 \\
\hline
\end{tabular}

Figure 24: Two 5+p RAID chains in each of four RAID arrays via two interfaces

\begin{tabular}{|c|c|c|c|c|}
\hline count & $\begin{array}{c}\text { block size } \\
(K B)\end{array}$ & $\begin{array}{c}\text { amount written } \\
(M B)\end{array}$ & $\begin{array}{c}\text { read rate } \\
(M B / s)\end{array}$ & $\begin{array}{c}\text { write rate } \\
(M B / s)\end{array}$ \\
\hline 5000 & 2048 & 10000 & 114.7 & 99.8 \\
\hline 10000 & 1024 & 10000 & 114.8 & 99.5 \\
\hline 20000 & 512 & 10000 & 114.9 & 99.4 \\
\hline 150000 & 64 & 9375 & 115.2 & 96.4 \\
\hline 600000 & 8 & 4688 & 77.0 & 68.1 \\
\hline 1000000 & $1 / 2$ & 488 & 5.6 & 5.3 \\
\hline
\end{tabular}

Figure 25: Two 5+p RAID chains in each of six RAID arrays via two interfaces 


\begin{tabular}{|c|c|c|c|c|}
\hline count & $\begin{array}{c}\text { block size } \\
(K B)\end{array}$ & $\begin{array}{c}\text { amount written } \\
(M B)\end{array}$ & $\begin{array}{c}\text { read rate } \\
(M B / s)\end{array}$ & $\begin{array}{c}\text { write rate } \\
(M B / s)\end{array}$ \\
\hline 100 & 512 & 0.05 & 0.5 & 0.5 \\
\hline 1000 & 512 & 0.5 & 1.7 & 0.7 \\
\hline 10000 & 512 & 5 & 1.7 & 1.2 \\
\hline 100000 & 512 & 51 & 1.4 & 1.2 \\
\hline 1000000 & 512 & 512 & 1.4 & 1.1 \\
\hline 10000000 & 512 & 5120 & 1.4 & 1.1 \\
\hline 100 & 8192 & 0.8 & 8.2 & 8.1 \\
\hline 1000 & 8192 & 8 & 16.3 & 8.1 \\
\hline 10000 & 8192 & 82 & 15.4 & 10.7 \\
\hline 100000 & 8192 & 819 & 16.1 & 10.5 \\
\hline 1000000 & 8192 & 8192 & 16.1 & 10.6 \\
\hline 100 & 65536 & 7 & 32.7 & 21.8 \\
\hline 1000 & 65536 & 66 & 21.8 & 23.4 \\
\hline 10000 & 65536 & 655 & 40.7 & 23.0 \\
\hline 100000 & 65536 & 6553 & 42.6 & 22.5 \\
\hline 100 & 524288 & 52 & 47.6 & 34.9 \\
\hline 1000 & 524288 & 524 & 51.9 & 29.2 \\
\hline 10000 & 524288 & 5242 & 53.7 & 27.7 \\
\hline 100 & 1048576 & 105 & 52.4 & 40.3 \\
\hline 1000 & 1048576 & 1048 & 51.6 & 29.2 \\
\hline 10000 & 1048576 & 10485 & 52.7 & 28.2 \\
\hline 100 & 2097152 & 210 & 51.1 & 34.3 \\
\hline 1000 & 2097152 & 2097 & 50.7 & 29.0 \\
\hline 10000 & 2097152 & 20971 & 50.6 & 28.7 \\
\hline & & & & \\
\hline
\end{tabular}

Figure 26: Varying the total amount of i/o to one 11+p RAID chain

\begin{tabular}{|c|c|c|c|c|c|}
\hline $\begin{array}{c}\text { number } \\
\text { of jobs }\end{array}$ & $\begin{array}{c}\text { block size } \\
(K B)\end{array}$ & $\begin{array}{c}\text { amount written } \\
(M B)\end{array}$ & $\begin{array}{c}\text { read rate } \\
(M B / s)\end{array}$ & $\begin{array}{c}\text { write rate } \\
(M B / s)\end{array}$ & $\begin{array}{c}\text { CPU } \\
\text { utilization }\end{array}$ \\
\hline 1 & 1048576 & 52600 & 15 & 14.6 & 0.2 \\
\hline 2 & 1048576 & 106721 & (no data) & 29.7 & 0.4 \\
\hline 4 & 1048576 & 88290 & (no data) & 24.5 & 0.6 \\
\hline 8 & 1048576 & 82924 & 23 & 23.0 & 2.0 \\
\hline
\end{tabular}

Figure 27: Similar tests run using the Ronnie benchmark on one $4+\mathrm{p}$ RAID chain 